\title{
Multiple phonemic restorations follow the rules for auditory induction
}

\author{
JAMES A. BASHFORD, JR., and RICHARD M. WARREN \\ University of Wisconsin, Milwaukee, Wisconsin
}

\begin{abstract}
Addition of noise to gaps produced by deletion of speech segments can result in both illusory continuity and increased intelligibility. Earlier findings suggest that the perceptual restoration of speech may represent a linguistic adaptation of a nonverbal phenomenon. "Auditory induction" permits perceptual synthesis of contextually appropriate sounds when deleted segments of a signal are replaced by a potential masker. In Experiment 1, when intelligible narrowband discourse was periodically interrupted by silence and by one of a number of bands of noise having different center frequencies, the illusory restoration of continuity was found to follow the spectral rules for nonverbal auditory induction. In Experiment 2, the durational limit of illusory continuity was determined for several types of broadband speech interrupted by broadband noise. The limiting duration was longest for normal discourse interrupted by noise and was equal to the average word duration of that stimulus. The results suggest that auditory induction becomes coupled with special linguistic mechanisms permitting perceptual synthesis of verbal components from noise, with the size of the segments dependent upon the extent of contextual information.
\end{abstract}

Under everyday listening conditions, signals of interest are often interrupted by other sounds. These interruptions can interfere seriously with perception of the signal. If they stimulate the cochlear units responding to the signal at a sufficiently high level, they can obliterate or mask auditory input corresponding to the signal. However, we possess a rather elegant mechanism that permits us to "hear" a verbal or nonverbal signal as intact despite obliteration of segments if the interruptions are brief. The restorative mechanisms producing this "temporal induction" employ information furnished by intact segments of the signal and are applied when, and only when, the power spectrum of the inducer admits the possibility that the restored sound could be present along with the louder sound.

The simplest type of temporal induction involves the apparent continuity of a fainter level $(A)$ when alternated with a louder level $\left(A^{\prime}\right)$ of the same sound $\left(A, A^{\prime}, A\right.$, $A^{\prime}$...). A somewhat more complex type of temporal induction involves illusory continuation of a sound such as a tone $(T)$ when alternated regularly with a louder sound of different spectral characteristics such as a broadband noise $(\mathrm{N})$ capable of masking the fainter sound were it

Portions of this work were supported by grants from the National Institutes of Health (5 R01 NS19295), the Air Force Office of Scientific Research (85-0260A), and the Graduate School of the University of Wisconsin-Milwaukee. We with to thank Albert S. Bregman and an anonymous reviewer for their helpful criticisms of an earlier version of this manuscript. These experiments were outlined in a paper presented at the 97th meeting of the Acoustical Society of America, with a brief abstract appearing in the Joumal of the Acoustical Society (vol. 65, Suppl. 1, 1979, S112), and an extended abstract appearing simultaneously in J. J. Wolf and D. J. Klatt (Eds.), Speech Communication Papers (New York: Acoustical Society of America, 1979, 423-426). The authors are both with the Department of Psychology at the University of Wisconsin, Milwaukee, WI 53201. actually present ( $T, N, T, N \ldots)$. In both these cases, auditory components corresponding to the continuous sound are subtracted from the louder inducer, so that the residual components of the inducer are heard as an intermittent addition to the continuous sound (see Warren, 1984).

The present study is concerned with temporal induction of speech. Unlike the other types of temporal induction described above, these "phonemic restorations" do not involve continuation of a steady-state signal, but rather, extrapolation and interpolation based on linguistic skills. In the first studies of phonemic restoration, it was demonstrated that a single fragment of a sentence corresponding to a phoneme or an entire syllable may be "heard" by listeners when replaced by a louder extraneous cough (Warren, 1970; Warren \& Obusek, 1971). Even after several presentations of the stimulus, listeners informed that a portion of the sentence was missing were typically unable to identify which speech sound (or sounds) was missing and could not localize the interrupting noise within the sentence. However, when a silent gap rather than a potential masking sound replaced the speech sound(s), the missing component could be identified and the gap located accurately. Phonemic restorations do not require coarticulation information provided by neighboring speech sounds, but represent a linguistically based completion, since the contextually appropriate sounds are restored even when the phoneme is mispronounced before its deletion and replacement by noise (Warren \& Sherman, 1974).

Phonemic restorations have been compared and related to nonverbal temporal induction (Warren, 1984; Warren, Obusek, \& Ackroff, 1972). However, phonemic restoration studies typically employ single interruptions, whereas nonverbal temporal induction studies typically employ multiple interruptions. The present study uses 
multiple interruptions of various types of speech with various types of sounds, and attempts to determine how linguistic context and spectral relations between the speech and extraneous sound influence illusory continuity. The conditions employed were selected to help clarify unresolved problems concerning perception of interrupted speech and to determine whether similar acoustic rules govern verbal and nonverbal temporal induction.

Before proceeding further, a brief historical review of the perceptual restoration of sounds is in order. The induced continuity of faint sounds interrupted by louder sounds was first reported as an incidental observation by Miller and Licklider (1950) in a study concerned primarily with the intelligibility of monosyllabic word lists periodically interrupted by silence. Miller and Licklider reported as an informal observation that interrupted monosyllables could be made to appear more natural and continuous by filling periodically spaced gaps in the words with a louder broadband noise. The effect observed by Miller and Licklider was limited to switching rates no lower than about $10 \mathrm{~Hz}$ (corresponding to noise and speech fragments no greater than $50 \mathrm{msec}$ ), and the enhanced continuity induced by interpolated noise was not accompanied by any increase in recognition accuracy. Perhaps for this reason, Miller and Licklider did not pursue a formal study of what they called the "picket fence" effect.

The preliminary observations of Miller and Licklider (1950) went unnoticed for many years, but illusory continuity effects have been rediscovered and studied independently in several laboratories (Houtgast, 1972; Thurlow, 1957; Vicario, 1960; Warren et al., 1972). The induction of continuity has proven to be a very general effect, but its duration depends upon the choice of signals serving as the inducer and inducee. For example, the illusory continuity of a broadband noise induced by a pure tone has been found to be limited to very brief gaps of from 5 to $15 \mathrm{msec}$ (Elfner, 1969; Elfner \& Caskey, 1965; Elfner \& Homick, 1966; Elfner \& Marsella, 1966), whereas the continuity of a narrowband noise induced by a louder broadband noise may persist through interruptions extending for tens of seconds (Warren et al., 1972). Illusory continuation of a tone interrupted periodically by a louder noise or a louder tone of different frequency has been observed to occur over durations of as long as 300 to 500 msec (Bregman \& Dannenbring, 1977; Warren et al., 1972).

Using nonverbal signals such as pure tones, both Houtgast (1972) and Warren et al. (1972) demonstrated a close correspondence between the ability of a louder sound to mask a fainter signal under simultaneous presentation and the ability of that sound to induce continuity of the fainter signal when they alternate in time. Thus, induction is greatest when stimulation by the inducer could correspond to masking of the inducee. On the basis of his observations, Houtgast (1972) considered the upper limit of induction (or, as he called it, "pulsation threshold") to be a measure of the inducing signal's spectral representation as projected from the cochlea, and he used illusory continuity to study possible lateral suppression effects in hearing (Houtgast, 1972, 1974a, 1974b). Although Houtgast (1972) and Warren et al. (1972) came to similar conclusions concerning the peripheral requirements for illusory continuity, because of the earlier discovery of the phenomic restoration effect, Warren et al. formulated the concept of temporal induction to encompass perceptual syntheses in addition to those leading to illusory continuance of steady-state sounds. It was considered that temporal induction of any sound could occur if it had sufficient probabilistic justification based on contextual information, and if the peripheral neural information admitted the possibility of masking by the louder sound. This theory has since been supported by studies with nonverbal signals demonstrating that temporal induction can produce not only the simple continuance of stationary signals such as pure tones, but also more complex restorations of distinct components within time-varying signals, including fragments of interrupted frequency glides (Dannenbring, 1976) and notes in familiar melodies that have been replaced by noise (Sasaki, 1980).

Studies involving auditory induction (phonemic restoration) for single interruptions of speech have shown that phonemes and whole syllables can be restored in keeping with the context provided by sentences and polysyllabic words (Layton, 1975; Obusek \& Warren, 1973; Samuel, 1981a, 1981b; Samuel \& Ressler, 1986; Sasaki, 1980; Warren, 1970; Warren \& Obusek, 1971; Warren \& Sherman, 1974). Although little is known about verbal induction that occurs under conditions of multiple interruption, indirect evidence suggests that the restoration of identifiable speech components can occur with multiple gaps, given sufficient contextual support. Although noise interpolation has not been found to improve the intelligibility of interrupted word lists (Dirks \& Bower, 1970; Miller \& Licklider, 1950), studies employing sentence and discourse stimuli have demonstrated an enhancement of intelligibility under conditions of periodic and aperiodic interruption (Cherry \& Wiley, 1967; Holloway, 1970; Powers \& Wilcox, 1977; Verschuure \& Brocaar, 1983). Furthermore, Powers and Wilcox (1977) have found that the noise-induced improvement in transcription accuracy for periodically interrupted sentences increased with noise amplitude up to a noise level $24 \mathrm{~dB}$ higher than the speech. The greatest increase in accuracy (about 20\%) at that noise level occurred when the interruption rate was $1.5 \mathrm{~Hz}$, corresponding to speech and noise fragments of $333 \mathrm{msec}$. Similar facilitation was subsequently obtained by Verschuure and Brocaar (1983) for both fully grammatical and semantically anomalous sentences.

The linguistic and acoustic factors found to influence the intelligibility of speech interrupted by noise are in keeping with the suggestion that temporal induction in the form of phonemic restoration can occur under conditions of multiple interruption. The effects of relative noise ampiitude on intelligibility (Powers \& Wilcox, 1977) are also consistent with the hypothesis that the restoration of in- 
telligibility is greatest when gaps in speech are filled with a potential masker (Warren \& Obusek, 1971). However, if temporal induction theory is valid, specifiable spectral as well as amplitude determinants of masking should be reflected in the restoration of absent speech segments.

Although the demonstrated correspondence between masking and phonemic restoration is less detailed to date than that for periodically interrupted nonverbal signals (Houtgast, 1972; Warren et al., 1972), there is relevant evidence available from studies using single interruptions of words and sentences. Layton (1975) and Samuel (1981b) found that the restoration of single phonemes is enhanced when deleted speech is replaced by a broadband sound such as noise or a recorded cough rather than by a pure tone. Using a signal detection paradigm, Samuel (1981a) also found that white noise produces greater restoration for the broadband sound (continuous spectra) of fricative speech components than for (the line spectra of) voiced speech sounds (Samuel, 1981b; Samuel \& Ressler, 1986). However, neither Layton nor Samuel and his co-worker recognized the role of temporal induction and masking in determining the extent of phonemic restoration.

While these studies dealing with single phonemic restorations are consistent with temporal induction theory, they do not provide a direct test of its validity. Experiment 1 of the present study was designed to test critically theoretical predictions concerning spectral relations of inducer and inducee using filtered speech (a prose passage) and filtered noise. The strength of restoration under various spectral conditions was measured in terms of the maximum duration of periodic gaps permitting illusory continuity. The same measure of induction strength was used in Experiment 2 with three different types of broadband speech (a recorded prose passage, the same prose passage read with words in reverse order, and monosyllabic word lists) to determine if the effects of a potential masker upon illusory continuity could be enhanced by contextual information.

\section{EXPERIMENT 1}

\section{Method}

Participants. The 20 listeners (11 women and 9 men ranging from 18 to 29 years of age) were enrolled in the introductory psychology course at the University of Wisconsin-Milwaukee. They were given either course credit or $\$ 1$ for their participation. They were selected from a larger pool of listeners on the basis of a hearing test carried out with a Békésy audiometer at least 1 day prior to their participation in the formal experiment.

Stimuli. The speech stimulus was a reading of continuous discourse excerpted from a magazine article on the Cuban missile crisis. The reading was produced in a sound-attenuating chamber (IAC Series 400 A) by a male speaker having a general American dialect. The output of an Altec microphone (Model 681A) was amplified by an Ampex AM-10 mixer, band-pass filtered from 100 to $8000 \mathrm{~Hz}$ with slopes of $48 \mathrm{~dB} /$ octave (Rockland Model 852 filter) and recorded on tape at $15 \mathrm{in} . / \mathrm{sec}$ using an Ampex 440-C eight- track recorder. During the recording of the passage, the speaker attempted to maintain a constant production rate by reading a wellrehearsed time-scored text while monitoring a digital readout of recording time. In an effort to minimize drifts in production level throughout the passage, the speaker also monitored his production using a General Radio Model 1565-A sound-level meter positioned directly beneath the recording microphone. To permit the speaker to more easily maintain a continuity of word rate, production level, and fundamental voicing frequency, the discourse stimulus was produced in $\mathbf{4 8}$ segments that averaged approximately 106 words in length. The total duration of the passage was $31 \mathrm{~min}$ and $10 \mathrm{sec}$, yielding an overall rate of $163 \mathrm{wpm}$. A panel of three trained listeners reviewed the recording and agreed that the reading was clearly articulated throughout, and that there were no obvious discontinuities in speech rate, loudness, or voicing frequency.

For a more detailed analysis of the discourse stimulus, 30 excerpts, each of 10-sec duration, were gated electronically at 1-min intervals from the recording and subjected to both peak level and pause duration analysis. Peak level measurement with a Brüel and Kjaer Model 2204 precision sound-level meter, operating in its impulse mode, yielded a range of $9.5 \mathrm{~dB}$ in peak levels with no consistent drift across the 30 excerpts. In order to estimate the average duration of speech components within the discourse recording, pause time was determined graphically with a General Radio Model 1521 B graphic level recorder, operating at a pen speed of 20 ips and a paper speed of $75 \mathrm{ipm}$. With pauses defined by the points where signal tracings fell to the level of inherent recording noise, the average percentage of pause time across excepts was $16.9 \%$. Subtraction of this estimated percentage pause time from the overall duration of the recording yielded an average word duration of $306 \mathrm{msec}$. On the basis of 20 sentences selected randomly from the text, the average syllabic composition was estimated to be 1.53 syllables per word. This yielded an estimated average syllabic duration of about $199 \mathrm{msec}$.

During the experiment, the discourse stimulus was subjected to on-line narrowband ( $1 / 8$-octave) filtering (Rockland Model 852 filter) with high-pass and low-pass slopes of $48 \mathrm{~dB} /$ octave intersecting at $1500 \mathrm{~Hz}$. This filtered stimulus (which was still intelligible to listeners unfamiliar with the passage) was interrupted on different trials by silence or by one of five 1/3-octave bands of pink noise. The pink noise (which has equal power per octave) was produced by a General Radio Model 1382 noise generator and passed through the tunable 1/3-octave filter of a Brüel and Kjaer Model 2121 frequency analyzer to produce bands with slopes of about $45 \mathrm{~dB} / \mathrm{oc}-$ tave and having center frequencies of $375,750,1500,3000$, and $6000 \mathrm{~Hz}$. These stimuli were prerecorded on the eight-track tape containing the discourse recording.

Apparatus. The narrowband noise and filtered discourse signals from the Ampex recorder were fed to separate subchannels of a Yamaha Model PM-430 eight-channel mixer, with noise and speech signals passed from separate master outputs of the mixer to separate electronic switches (Grason-Stadler Model 1287-B). The two switches were set for $10 \mathrm{msec}$ rise/fall and were triggered alternately, with a $50 \%$ duty cycle, by a Grason-Stadler Model 1219 sequence counter, driven by logic pulses from a Hunter Model 1516 interval timer. The pulse rate of the interval timer was controlled by the frequency of a sinusoidal tone produced by a Wavetek Model 135 tone generator. During the experiment, this tone generator, with its dial concealed, was used by listeners to vary the rate (duration) of speech interruption. The frequency scale of the oscillator dial and the counting cycle of the interval timer were adjusted so that variation in the dial's position produced a continuous variation of interruption rate proportional to the angular position of the control knob over a range of .5 to 25 ips. The corresponding durations of speech off-time and on-time during each cycle of interrup- 
tion ranged from $1 \mathrm{sec}$ to $20 \mathrm{msec}$, and could be measured with an accuracy of $.01 \mathrm{msec}$ by a Hewlett-Packard universal counter/timer (Model 5302A).

The alternately gated signals from the two electronic switches were combined with a Grason-Stadler Model 1292 passive mixer, and the resulting signal was passed through an impedance-matching transformer (Grason-Stadler Model E10589A) and finally transduced diotically through a matched pair of Telephonics TDH-49 headphones mounted in MX 41/AR cushions. Stimuli were presented at a peak speech amplitude and average noise amplitude of $80 \mathrm{dBA}$ SPL.

Procedure. At least 1 day after audiometric screening (which excluded not only listeners with hearing impairments, but also those who did not follow the standard audiometric instructions), participants were tested individually in the formal experiment. They were told that they would be listening to a recorded voice and that their task would be to adjust a dial to the point at which interruptions in the speech signal were just clearly detectable. During the instruction phase of the experiment, listeners were presented with a 10-sec sample of uninterrupted discourse to acquaint them with the stimulus and to ensure that they would distinguish between intentional pauses produced by the speaker and the externally imposed interruptions that they would control during the experiment. Listeners were encouraged to explore the entire range of dial settings in making their judgments, but it was emphasized that they should always search for the dial setting at which interruption was just clearly detectable.

The oscillator was positioned so that its dial was easily adjustable but concealed from the subject, and the dial's starting position for each judgment was at the highest interruption rate of 25 ips (ontime $=$ off-time $=20 \mathrm{msec}$ ). Each listener made a total of 30 interruption threshold adjustments across five blocks of trials. Within each block, an adjustment was made with each of the five noise bands and with silence serving as the interrupter. The order in which these six conditions appeared within blocks was pseudorandomized for each listener, with the restriction that no condition appear twice in succession across blocks.

Listeners were given as much time as needed to make their threshold adjustments. The tape location reached at the end of each trial served as the beginning point for the following trial. The experimenter recorded the off-time corresponding to the listener's chosen interruption rate (measured by the Hewlett-Packard timer) and reset the listener's oscillator for each new trial. Listeners averaged about $30 \mathrm{sec}$ per judgment and the average intertrial interval was approximately $10 \mathrm{sec}$. The average duration of an experimental session, including instruction and debriefing, was about $35 \mathrm{~min}$.

\section{Results and Discussion}

The median off-time for a listener's five adjustments with each interrupter was treated as their interruption threshold for that stimulus. The means of those median scores are presented in Table 1 .

A treatments $X$ subjects analysis of variance performed upon listeners' threshold scores yielded a significant interrupter effect $[F(5,95)=14.59, p<.001]$. Subsequent Newman-Keuls analysis indicated that the threshold interruption duration was greatest $(p<.01)$ when the band-pass speech was interrupted by the noise band having the same $(1500 \mathrm{~Hz})$ center frequency. The next greatest threshold duration ( $p<.05$ or better) was obtained with the noise band centered at $750 \mathrm{~Hz}$. Interruption thresholds in the remaining conditions, including interruption by silence, did not differ significantly.
Table 1

Mean Median Interruption Thresholds (in Milliseconds Off-Time) for Narrowband Speech (1500 Hz Center Frequency) Interrupted by Narrowband Noise Having Center Frequencies Listed Below

\begin{tabular}{llllll}
\hline Noise Band (Hz) & 375 & 750 & 1500 & 3000 & 6000
\end{tabular}

$\begin{array}{llllll}\text { Thresholds (msec) } & 135.7 & 220.7 & 304.1 & 129.2 & 127.9\end{array}$

Note--The threshold off-time with silence substituted for the interpolated noise bands was $79.2 \mathrm{msec}$.

The results obtained are consistent with the theory offered by Warren and his co-workers (Warren, 1982, 1984; Warren et al., 1972), which considers auditory induction (including phonemic restoration) to be a process that routinely compensates for intermittent masking by employing the neural input produced by an extraneous noise for abstracting components corresponding to a contextually likely signal. The greatest induction of continuity for the filtered discourse $(304 \mathrm{msec}$ ) occurred when the speech and noise bands were matched in center frequency, and was influenced asymmetrically by the nonmatching noise bands. The average threshold off-time was $221 \mathrm{msec}$ for the noise band centered one octave below the speech band, and was $129 \mathrm{msec}$ for the noise band centered one octave above. A similar asymmetry or "upward spread" occurs in masking, perhaps due to the asymmetrical way in which frequency information is encoded at the cochlea (Wegel \& Lane, 1924), and this asymmetry has also been observed for illusory continuity of interrupted fixedfrequency tones (Houtgast, 1972; Warren et al., 1972).

The maximum duration of induction for the prose passage in this experiment is much greater than that reported by Miller and Licklider (1950) for word lists, suggesting that the threshold duration of interruption may prove to be a useful measure of contextual factors governing verbal induction (this suggestion was tested in Experiment 2). The results also suggest that the limiting gap duration of induction of discourse may be related to the duration for linguistic units within the stimulus. The threshold gap duration of $304 \mathrm{msec}$ found with the most effective band of interpolated noise (i.e., the noise band with the same center frequency as the speech band) was almost exactly equal to the average word duration in the passage (306 msec). Experiment 2 was designed to replicate this observation and to further examine the contextual limits of verbal induction using additional, less contextually bound, speech stimuli.

\section{EXPERIMENT 2}

In addition to the recorded discourse passage used in Experiment 1, a list of isolated monosyllabic words was presented to listeners in an attempt to replicate the weak continuity effect reported for that stimulus by Miller and Licklider (1950). A second "low-context" condition, which served as a control for the discourse stimulus, consisted of the same discourse passage read backward as prose (i.e., the passage was read starting with the last 
word and progressing to the first). This reversal of word order preserved the lexical components and the physical continuity of the stimulus, but destroyed the higher order syntactic and semantic content of the original passage. The major purpose of the experiment was to compare the effects of interpolated noise and silence upon induction thresholds for these three types of speech. However, normal discourse was also paired with a pure tone internupter for comparison with results from earlier studies dealing with the restoration of single phonemes.

\section{Method}

Participants. A second group of 20 students (12 women and 8 men ranging from 18 to 27 years of age) served as listeners in this experiment. All subjects passed the audiometric screening task used for selection of listeners in Experiment 1.

Stimuli. The additional speech stimuli were produced by the same speaker using the same apparatus and monitoring procedures employed in Experiment 1. For production of the backward reading of discourse, the original passage was retyped with the order of words reversed and with sentential grouping preserved. The speaker attempted to read the text as prose, and produced the passage in segments identical to those used in the production of the forward reading. The elapsed time for the forward reading of each segment served as the speaker's target production time for the duration of its backward counterpart. The monitoring procedure did permit a relatively close match in overall production rates for the two discourse recordings. The total duration of the forward reading was $31 \mathrm{~min}$ and $10 \mathrm{sec}$, yielding an overall rate of $163 \mathrm{wpm}$; the duration of the backward reading was $31 \mathrm{~min}$ and $39 \mathrm{sec}$, yielding a rate of about $161 \mathrm{wpm}$. Thirty 10 -sec excerpts were gated from the backward discourse recording at 1-min intervals and subjected to peak level and pause duration analysis. Peak levels across the 30 excerpts of backward discourse covered a range of about $12.5 \mathrm{~dB}$. Pause time was determined graphically using the same procedure and criteria applied to the forward discourse reading. The average percentage of pause time across excerpts was somewhat less for the backward reading $(8.6 \%)$ than for the forward reading $(16.9 \%)$. Correspondingly, the estimated average duration of speech components was greater for the backward reading $(341 \mathrm{msec} /$ word; $222 \mathrm{msec} / \mathrm{syllable}$ ) than for the forward discourse reading (306 msec/word; $199 \mathrm{msec} / \mathrm{syllable}$ ). ${ }^{1}$

The remaining speech stimulus was a list of 500 phonetically balanced (PB) monosyllabic words, consisting of the first 10 standard 50-word lists (American National Standards Institute 53.2-1960 [R19710]). The words were produced at a rate (including pauses between words) of .33 words/sec. Peak levels varied less than $9 \mathrm{~dB}$ across the 500 words. For estimation of average monosyllable duration, 5 words were selected at random from each of the 10 lists (a total of 50 words sampled), and these sampled words were subjected to graphic level analysis. With word onsets and offsets defined by the points where signal tracings fell to the level of inherent recording noise, the average word duration was approximately $583 \mathrm{msec}$.

The backward discourse and word list recordings were subject to evaluation by the same panel of listeners who reviewed the normal discourse recording. The panel agreed that the additional rocordings were pronounced clearly throughout, and that there were no obvious discontinuities in production rate, loudness level, or voicing frequency during either recording.

During the experiment, all speech signals were presented broadband (band-pass filtering limits of 100 and $8000 \mathrm{~Hz}$ with slopes of $48 \mathrm{~dB} /$ octave) and were interrupted on different trials by silence, a 2-kHz sinusoidal tone, or by a band of pink noise having the same bandwidth and filtering slopes as the speech.

The apparatus differed little from that used in Experiment 1. The three speech signals from the Ampex recorder were passed directly to the Yamaha mixer and could be selectively passed through the master "speech" channel output of the mixer to one of the two electronic switches. A 2-kHz sinusoidal tone and broadband pink noise (produced on-line and band-pass filtered from 100 to $8000 \mathrm{~Hz}$ with slopes of $48 \mathrm{~dB} /$ octave) could be passed through the remaining "nonspeech" output channel of the mixer to the remaining electronic switch. When the nonspeech channel was inactive, the speech signals were interrupted by silence. As in Experiment 1, the rate of speech interruption (with a $50 \%$ duty cycle and $10 \mathrm{msec}$ rise/decay) was controlled by the listener. The stimuli were again presented through diotically wired headphones, with the peak amplitude of speech stimuli and the average amplitude of the tone and noise at 80 dBA SPL.

Procedure. Listeners were given essentially the same instructions used in Experiment 1 and were also presented with uninterrupted 10-sec samples of each speech stimulus prior to testing. Each listener made a total of 21 internuption threshold adjustments across three blocks of trials. Within each block, an adjustment was made for each of the three speech stimuli (normal discourse, backward discourse, and isolated monosyllables) internupted both by silence and by noise. In addition, the normal discourse stimulus was also interrupted by a $2-\mathbf{k H z}$ tone. The order in which these seven conditions appeared within blocks for each listener was pseudorandomized with the restriction that no condition appear twice in succession across blocks. As in Experiment 1, listeners were given as much time as needed to make their threshold adjustments, with the tape location reached at the end of each trial for a particular speech stimulus serving as the beginning point for the following trial with that stimulus. Listeners averaged about 1 min per judgment, and the average intertrial interval was approximately $30 \mathrm{sec}$. The average duration of an entire session, including instruction and debriefing, was about $45 \mathrm{~min}$.

\section{Results}

The median off-time for a listener's three adjustments with each combination of speech and interrupter stimuli was treated as their interruption threshold for that condition. The means of those median scores for the 20 listeners are presented in Table 2 . Table 3 presents the estimated average durations of words, syllables, and phonemes within each speech stimulus, based upon a random sampling of 20 sentences from the discourse text and 50 monosyllables from the PB lists.

Listeners' threshold scores for the conditions with interpolated noise and silence were subjected to a treatments

Table 2

Mean Median Interruption Thresholds (in Milliseconds Off-Time) for each Combination of Interrupter and Speech Stimulus

\begin{tabular}{|c|c|c|c|}
\hline \multirow[b]{2}{*}{$\begin{array}{l}\text { Internupting } \\
\text { Stimulus }\end{array}$} & \multicolumn{3}{|c|}{ Speech Stimulus } \\
\hline & $\begin{array}{c}\text { Normal } \\
\text { Discourse }\end{array}$ & $\begin{array}{l}\text { Backward } \\
\text { Discourse }\end{array}$ & $\begin{array}{c}\text { Isolated } \\
\text { Monosyllables }\end{array}$ \\
\hline Noise & 304.4 & 147.7 & 161.5 \\
\hline Silence & 52.0 & 50.0 & 61.1 \\
\hline Tone & 72.1 & & \\
\hline
\end{tabular}

Note-The tonal interrupter was paired only with normal discourse. 
Table 3

Average Durations (in Milliseconds) of Components Within the Speech Stimuli Presented in Experiment 2

\begin{tabular}{|c|c|c|c|}
\hline \multirow[b]{2}{*}{ Components } & \multicolumn{3}{|c|}{ Speech Stimulus } \\
\hline & $\begin{array}{c}\text { Normal } \\
\text { Discourse }\end{array}$ & $\begin{array}{l}\text { Backward } \\
\text { Discourse }\end{array}$ & $\begin{array}{c}\text { Isolated } \\
\text { Monosyllables }\end{array}$ \\
\hline Words & 306 & 341 & 583 \\
\hline Syllables & 199 & 222 & 583 \\
\hline Phonemes & 78 & 87 & 178 \\
\hline
\end{tabular}

$x$ treatments $x$ subjects analysis of variance. The main effects of speech type $[F(2,38)=18.83]$ and interrupter $[F(1,19)=30.80]$ and the interaction of those two factors $[F(2,38)=14.81]$ were each significant at the .001 level. Subsequent Newman-Keuls analysis revealed that, whereas thresholds for interruption by silence did not differ across the three speech conditions, thresholds for noise-filled interruption were higher $(p<.01)$ for the normal reading of discourse than for isolated monosyllables or for discourse read backward. Thresholds for the two low-context stimuli interrupted by noise did not differ. Planned orthogonal comparisons between silence and noise-filled interruption thresholds within speech types indicated that noise insertion raised thresholds both for normal discourse $[t(19)=5.39, p<.001]$ and for monosyllables $[t(19)=2.14, p<.05]$. The difference in thresholds with interpolated noise versus silence for the backward reading of discourse was of doubtful reliability $[t(19)=2.09, p<.10]$.

The threshold scores for forward discourse, including those from the pure tone interrupter condition, were also subjected to a separate treatments $\times$ subjects analysis of variance that yielded a significant interrupter effect $[F(2,38)=27.98, p<.001]$. Subsequent NewmanKeuls analysis indicated that interruption thresholds were higher with interpolated noise than with tone or silence $(p<.01)$. The difference between the tone-filled and silence-filled conditions was significant only by $t$ test $[t(19)=2.17, p<.05]$.

\section{Discussion}

The weak induction effect produced by the tonal interrupter in this experiment is consistent with the findings of Layton (1975) and Samuel (1981b) for the restoration of single phonemes, but is much more pronounced under the present conditions of multiple restoration. Interruption thresholds increased by only $20 \mathrm{msec}$ when silent gaps in the discourse were filled with the tone, compared with an increase of $252 \mathrm{msec}$ when noise was used. This small effect of tone on speech continuity is comparable in magnitude to that observed for a tone serving as a periodic inducer of broadband noise (Elfner \& Caskey, 1965).

As predicted, interruption thresholds were far higher with a broadband interpolated noise serving as an inducer, and varied as a function of context. The average interruption threshold for normal discourse with interpolated noise was almost exactly equal to the average word duration (corresponding to about 1.5 syllables). It should be noted that this correspondence between interruption threshold and word length was also found in Experiment 1 for narrowband speech interrupted by a slightly broader band of noise having the same center frequency. In contrast, interruption thresholds corresponded to only about $43 \%$ of the average word duration and to $67 \%$ of the average syllable duration in the backward discourse reading. The interruption threshold for isolated words was of approximately the same duration as for the backward reading of discourse, but corresponded to only about $28 \%$ of the average isolated monosyllable.

The relatively small induction effect found for both discourse read backward and for isolated words supports the hypothesis that the high thresholds observed in both experiments for the normal (forward) discourse reading were due to the additional syntactic and semantic context provided by the original passage, and not simply to its lexical content or acoustic continuity. In fact, the forward reading of the prose passage, having proportionately more pause time, was acoustically less continuous than the backward reading.

The low durational limit found for induction of word lists in the present study is consistent with the conclusions of Dirks and Bower (1970) and Miller and Licklider (1950), who reported that noise interpolation under conditions of periodic interruption did not improve the intelligibility of isolated monosyllabic words. In contrast, the interruption threshold found for discourse indicates that induction of connected speech occurred at durations of interruption previously found to permit the restoration of intelligibility for syntactically lawful but semantically anomalous "sentences," such as "The church bell drank a sailor" (Verschuure \& Brocaar, 1983), and for syntactically and semantically acceptable sentences (Powers \& Wilcox, 1977; Verschuure \& Brocaar, 1983). Furthermore, the threshold gap duration for discourse interrupted by noise in the present study $(304 \mathrm{msec})$ is also close to that $(333 \mathrm{msec})$ found by Powers and Wilcox (1977) to permit the greatest restoration of sentence intelligibility.

\section{CONCLUSIONS}

The results of the present study indicate that the periodic interruption paradigm represents a useful "statistical" approach to the study of phonemic restoration. By presenting the listener with a heterogeneous sampling of regular interruptions uncorrelated with events within the speech signal, the technique offers both generality of results and a sensitivity to some factors less easily studied under conditions of single interruption. One case in point is the detailed correspondence between masking and verbal induction found in Experiment 1. As assumed by Houtgast (1972) and Warren et al. (1972), both masking and induction require for their occurrence an overlap in 
the neural patterns of stimulation produced by the signal and noise. This is consistent with the suggestion by Warren et al. (1972) and by Warren and Sherman (1974) that the neural activity produced by the interrupting noise is used as the substrate for constructing the restored fragment in auditory induction (including phonemic restoration), thus ensuring that perceptual synthesis is restricted to sounds that might actually have been obliterated by an interpolated masker (see also Warren, 1976, p. 403). Samuel (1981b), unaware of this earlier work, stated that "overall spectral similarity" is the most potent acoustic factor governing phonemic restoration, thus coming close to discovering independently the role of masking in this phenomenon.

The present results also provide additonal support for the suggestion (Warren \& Obusek, 1971) that multiple phonemic restorations might be responsible for previous observations of the enhanced intelligibility produced by adding noise to the gaps in interrupted speech. The variation of induction thresholds across the different types of speech presented to listeners in Experiment 2 is in keeping with contextual factors previously found to govern the noise-induced enhancement of intelligibility (Cherry \& Wiley, 1967; Holloway, 1970; Powers \& Wilcox, 1977; Verschuure \& Brocaar, 1983). In addition, preliminary results in this laboratory with filtered versions of the same (CID) sentences used in broadband form by Powers and Wilcox (1977) indicate that the interpolation of narrowband noises produces spectrally dependent effects upon intelligibility similar to those found to govern illusory continuity in Experiment 1.

The correspondence between the induction duration limits in Experiment 2 (broadband discourse alternated with broadband noise) and Experiment 1 (narrowband discourse alternated with spectrally matched narrowband noise) indicates that this limiting value for discourse is robust. Also, the wide variation in continuity limits under different conditions (from 50 msec with silent gaps up to $300 \mathrm{msec}$ with spectrally matching noise) suggests that there is a sufficient range of values to permit useful measurements of factors influencing the extent of illusory continuity under a variety of conditions. It is interesting to note that whereas the upper limit of induction for discourse (consisting of a normal mix of monosyllabic and polysyllabic words) was reached when periodic switching passed on average just one fragment per word, with a passage having the same words read in reversed order the limit dropped to one fragment per syllable and with isolated monosyllables it decreased further to about one fragment per phoneme. These results suggest that the durational limits of continuity, as measured in the present study, may reflect the size of units employed for perceptual organization of speech under various contextual constraints.

\section{REFERENCES}

Bregman, A. S., Dannenbring, G. L. (1977). Auditory continuity and amplitude edges. Canadian Journal of Psychology, 31, 151-159.
Cherry, C., \& WiLEY, R. (1967). Speech communications in very noisy environments. Nature, 214, 1164.

Dannenbring, G. L. (1976). Perceived auditory continuity with alternately rising and falling frequency transitions. Canadian Journal of Psychology, 30, 99-114.

Dirks, D. D., \& BowER, D. (1970). Effect of forward and backward masking on speech intelligibility. Journal of the Acoustical Society of America, 47, 1003-1007.

ELFNER, L. F. (1969). Continuity in alternately sounded tone and noise signals in a free field. Journal of the Acoustical Society of America, 46, 914-917.

Elfner, L. F., Caskey, W. E. (1965). Continuity effects with alternately sounded noise and tone signals as a function of manner of presentation. Journal of the Acoustical Society of America, 38, 543-547.

ELFNER, L. F., \& Homick, J. L. (1966). Some factors affecting the perception of continuity in alternately sounded tone and noise signals. Journal of the Acoustical Society of America, 40, 27-31.

ElfNer, L. F., \& MARsella, A. J. (1966). Continuity effects with alternately sounded noise and tone signals. Medical Research \& Engineering, 5, 22-23.

Holloway, C. M. (1970). Passing the strongly voiced components of noisy speech. Nature, 226, 178-179.

Houtgast, T. (1972). Psychophysical evidence for lateral inhibition in hearing. Journal of the Acoustical Society of America, 51, 1885-1894.

Houtgast, T. (1974a). Masking patterns and lateral inhibition. In E. Zwicker \& E. Terhardt (Eds.), Facts and models in hearing (pp. 258-265). Berlin: Springer-Verlag.

Houtgast, T. (1974b). The slopes of masking patterns. In E. Zwicker \& E. Terhardt (Eds), Facts and models in hearing (pp. 269-272). Berlin: Springer-Verlag.

LAyTON, B. (1975). Differential effects of two nonspeech sounds on phonemic restoration. Bulletin of the Psychonomic Society, 6, 487-490.

Milier, G. A., \& Licklider, J. C. R. (1950). The intelligibility of interrupted speech. Journal of the Acoustical Society of America, 22, 167-173.

OBusex, C., \& WARREN, R. M. (1973). Relation of the verbal transformation and the phonemic restoration effects. Cognitive Psychology, 5, 97-107.

Powers, G. L., \& Wilcox, J. C. (1977). Intelligibility of temporally interrupted speech with and without intervening noise. Journal of the Acoustical Society of America, 61, 195-199.

SAmUeL, A. G. (1981a). Phonemic restoration: Insights from a new methodology. Journal of Experimental Psychology: General, 110, 474-494.

SAMUEL, A. G. (1981b). The role of bottom-up confirmation in the phonemic restoration illusion. Journal of Experimental Psychology: Human Perception \& Performance, 7, 1124-1131.

SAMUel, A. G., \& Ressler, W. H. (1986). Attention within auditory word perception: Insights from the phonemic restoration illusion. Journal of Experimental Psychology: Human Perception \& Performance, 12, 70-79.

SASAKI, T. (1980). Sound restoration and temporal localization of noise in speech and music sounds. Tohuku Psychologica Folia, 39, 79-88.

ThurLow, W. R. (1957). An auditory figure-ground effect. American Journal of Psychology, 70, 653-654.

VERSCHUURE, J., \& BROCAAR, M. P. (1983). Intelligibility of interrupted meaningful and nonsense speech with and without intervening noise. Perception \& Psychophysics, 33, 232-240.

Vicario, G. (1960). L'effetto tunnel acustico. Rivista di Psicologia, 54, 41-52.

WARREN, R. M. (1970). Perceptual restoration of missing speech sounds. Science, 167, 392-393.

WARREN, R. M. (1976). Auditory illusions and perceptual processes. In N. J. Lass (Ed.), Contemporary issues in experimental phonetics (pp. 389-417). New York: Academic Press.

WARREN, R. M. (1982). Auditory perception: A new synthesis. New York: Pergamon Press.

WARREN, R. M. (1984). Perceptual restoration of obliterated sounds. Psychological Bulletin, 96, 371-383. 
WARREN, R. M., \& OBUSEK, C. J. (1971). Speech perception and phonemic restorations. Perception \& Psychophysics, 9, 358-362.

WarRen, R. M., OBuSEK, C. J., \& ACKRofF, J. M. (1972). Auditory induction: Perceptual synthesis of absent sounds. Science, 176, 1149-1151.

Warren, R. M., \& Sherman, G. L. (1974). Phonemic restorations based on subsequent context. Perception \& Psychophysics, 16, 150-156.

WegEL, R. L., \& LANE, C. E. (1924). The auditory masking of one pure tone by another and its probable relation to the dynamics of the inner ear. Physical Review, 23, 266-285.

\section{NOTE}

1. Reading of a syntactically and semantically anomalous passage was a difficult task, and the speaker's matching of overall production rate to that of normal discourse was accomplished in part through a reduction in pause time and a corresponding $11 \%$ expansion of component durations for the backward reading.

(Manuscript received September 2, 1986; revision accepted for publication February 1, 1987) 